ARTICLE

\title{
A novel mechanism for the loss of mRNA activity in lipid nanoparticle delivery systems
}

\author{
Meredith Packer ${ }^{1}$, Dipendra Gyawali ${ }^{1}$, Ravikiran Yerabolu ${ }^{1}$, Joseph Schariter ${ }^{1} \&$ Phil White (i) ${ }^{1 凶}$
}

Lipid nanoparticle (LNP)-formulated mRNA vaccines were rapidly developed and deployed in response to the SARS-CoV-2 pandemic. Due to the labile nature of mRNA, identifying impurities that could affect product stability and efficacy is crucial to the long-term use of nucleic-acid based medicines. Herein, reversed-phase ion pair high performance liquid chromatography (RP-IP HPLC) was used to identify a class of impurity formed through lipid:mRNA reactions; such reactions are typically undetectable by traditional mRNA purity analytical techniques. The identified modifications render the mRNA untranslatable, leading to loss of protein expression. Specifically, electrophilic impurities derived from the ionizable cationic lipid component are shown to be responsible. Mechanisms implicated in the formation of reactive species include oxidation and subsequent hydrolysis of the tertiary amine. It thus remains critical to ensure robust analytical methods and stringent manufacturing control to ensure mRNA stability and high activity in LNP delivery systems.

\footnotetext{
${ }^{1}$ Moderna, Inc., 200 Technology Square, Cambridge, MA, USA. ${ }^{凶}$ email: phil.white@modernatx.com
} 
$\mathrm{N}$ ucleic acid-based medicines have emerged as promising alternatives to more traditional vaccines and therapeutics ${ }^{1,2}$. Most notably, mRNA-based vaccines recently developed by Pfizer/BioNTech and Moderna changed the course of the SARS-CoV-2 pandemic, receiving swift emergency use authorization for public use due to efficacy and safety demonstrated in phase 3 trials $^{3,4}$. The rapid deployment of these vaccines was in part due to the advantages offered by mRNA versus conventional vaccines, including flexibility of mRNA sequence design and scalability of the manufacturing process. Furthermore, the rapid biodegradability of mRNA makes it an appealing modality from a safety and pharmacokinetic perspective $^{5}$; however, this same intrinsic instability is a key shelflife limiting parameter and hurdle to effective vaccine delivery through various routes of administration ${ }^{6}$.

The effective delivery of mRNA-based vaccines and therapies is enabled by the use of lipid nanoparticles (LNPs), which protect nucleic acid degradation by exo- and endonucleases ${ }^{7,8}$ and facilitate cellular uptake and expression ${ }^{9,10}$. Used in both the Pfizer/BioNTech and Moderna COVID-19 mRNA vaccines, this delivery system is particularly effective as it leverages LNP surface properties $^{11-14}$, the ability of LNPs to facilitate endosomal escape through ionization of the amino lipid ${ }^{15,16}$, and tissue-specific mRNA delivery based on particle size ${ }^{17}$. Together, these features improve vaccine immunogenicity ${ }^{18}$.

Although LNP technology is an effective route for mRNA delivery to tissues, the interaction of certain chemical functionalities during storage such as oxidation, hydrolysis, or transesterification can lead to mRNA degradation ${ }^{19,20}$ through backbone cleavage of the mRNA into smaller fragments ${ }^{21}$.

Herein we report the discovery, characterization, and identification of another class of mRNA reactivity that leads to loss in activity: the formation of lipid-mRNA adducts through the covalent addition of reactive lipid species to the nucleobase. Importantly, as many lipid-based nucleic acid formulations share common chemical functionalities, particularly those that use an ionizable amino-lipid, mechanisms identified in this study are broadly applicable. These data can inform manufacturing protocols to limit the formation of lipid-mRNA adducts and ensure the high quality of nucleic acid-based products.

\section{Results}

Discovery of lipid-modified mRNA species in formulated mRNA-LNP system. Reversed phase-ion pair high performance liquid chromatography (RP-IP HPLC) is a well-accepted method alongside agarose or polyacrylamide gel or capillary electrophoresis (CE) to assess mRNA integrity ${ }^{22,23}$. As in more conventional RP chromatography modes, separation of phases is driven by hydrophobic interactions between the analyte and stationary phase; however, since the phosphodiester mRNA backbone is highly polar, high salt concentrations are necessary to neutralize the negative charge and allow retention based on hydrophobicity of the aromatic nucleobases. Alkylammonium salts can increase hydrophobic interactions, driving selectivity based on the number of charges conferred by sequence length and enabling high resolution size-based separations (Fig. 1a). This provides a similar separation to $\mathrm{CE}$, which is driven by size and charge; however, based on the ion pair system used, RP-IP retains some selectivity to variations in mRNA hydrophobicity due to sequence or chemical modifications ${ }^{24}$.

When RP-IP HPLC integrity analysis was applied to mRNA extracted from an mRNA-LNP, a late eluting-peak (LP) was detected by HPLC (Fig. 1b) that was not observed by CE (Fig. 1c). The elution time for the LP was $21 \mathrm{~min}$, distinct from the mRNA elution time of $10-16 \mathrm{~min}$ (Fig. 1b). The ultraviolet (UV) spectrum of the LP had the same maximum absorbance at $260 \mathrm{~nm}$ as the mRNA main peak (MP), confirming the LP to be an RNA-related population (Fig. $1 \mathrm{~b}$ inset). Equivalent levels of the LP were observed under various analytical conditions, including modified RNA extraction, RP-IP mobile phase conditions, and stationary phase selection and temperature, suggesting it was not a separation artifact.

To further understand the late eluting time of the LP compared with various-length mRNAs, modified gradient conditions were applied, revealing the LP to be a heterogenous mixture of resolved, well-defined peaks with a characteristic fingerprint (Fig. 1d). Higher levels of the LP from mRNA-LNP formulations were observed over time at higher storage temperatures (Fig. 1e).

Characterization of the modified mRNA fraction. mRNA was extracted from LNPs and fractionated by RP-IP HPLC to generate purified MP and LP fractions (Supplementary Fig. 1). Upon reanalysis, separation of the MP and LP was preserved when assessed by RP-IP HPLC (Fig. 2a), but fractions were not distinguished by CE (Fig. 2b).

To investigate whether tertiary mRNA structures played a role, size exclusion chromatography (SEC) at ambient conditions was applied to the MP and LP. The extensive intra- and intermolecular structure of mRNA molecules leads to a sequence-, salt, and temperature-dependent ensemble of structures; these are typically denatured under RP-IP HPLC or CE conditions but can be resolved by native $\mathrm{SEC}^{25}$. SEC analysis revealed that the MP and LP fraction profiles were identical to mRNA extracted from the LNP, with a dominantly monomeric profile (Fig. 2c). Together, these results eliminated aggregation as the origin of the LP and strongly implicate additional hydrophobicity behind this phenomenon.

We next applied compositional analysis to differentiate the LP from MP. The UV spectral data did not distinguish LP from MP (Fig. 1b). Fourier-transform infrared spectroscopy (FT-IR) revealed minor differences potentially consistent with chemical modification (Supplementary Fig. 2). Next-generation sequencing (Supplementary Fig. 3) and RNA oligonucleotide mapping by digest-based mass spectrometry (MS; Supplementary Fig. 4) showed an identical profile between the MP and LP, suggesting non-site-specific modifications with low abundance. Nucleotide profiling by LC-UV showed identical composition of nucleobases (Supplementary Fig. 5); MS analysis did not identify LP modifications, potentially due to poor ionization of lipid-modified nucleotide species in negative electrospray ionization mode.

Identification of lipid-modified nucleosides. Nucleoside profiling was performed by enzymatic digestion of the LP and MP fractions and analysis using positive mode LC-MS/MS. Although UV and total ion-current chromatograms showed identical composition of 4 unmodified nucleobases for the MP and LP fractions, differential analysis revealed several mass-to-charge $(\mathrm{m} / \mathrm{z})$ values exclusively found in the isolated LP, with $<1 \%$ abundance relative to total unmodified nucleosides (Fig. 3a). Under LC conditions used, elution time of these unique masses was 9.5-11 min; this elution time was later compared with unmodified nucleosides (2-8 $\mathrm{min})$ and earlier compared with ionizable lipid (12 min), indicating the unique masses had an intermediate hydrophobicity between the unmodified nucleosides and ionizable lipid.

To elucidate the structure of each reacted nucleoside detected, elemental composition was assigned based on high-resolution MS data. Further MS/MS analyses across reacted nucleosides showed covalent addition of additional mass via the nucleobase, as shown by the fragmentation pattern of the ion at $\mathrm{m} / \mathrm{z} 526.30$ (Fig. 3b). The presence of a fragment ion at $\mathrm{m} / \mathrm{z} 112.05$ corresponds to the 
a

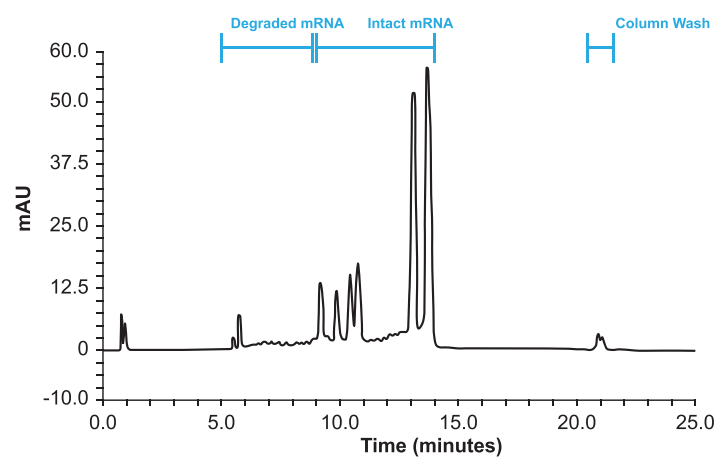

C

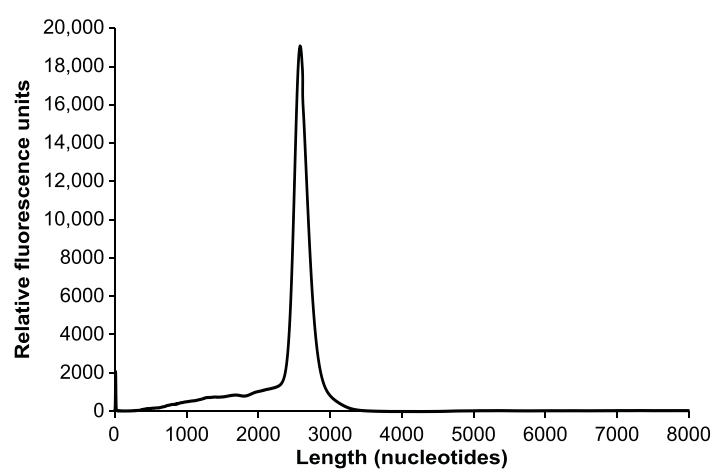

b

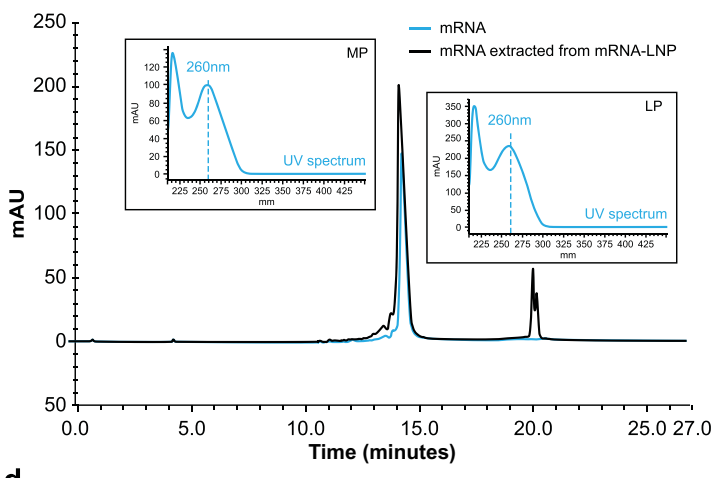

d

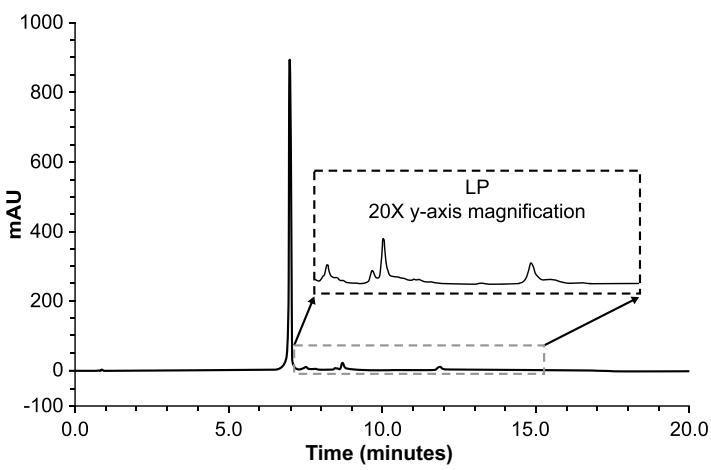

e

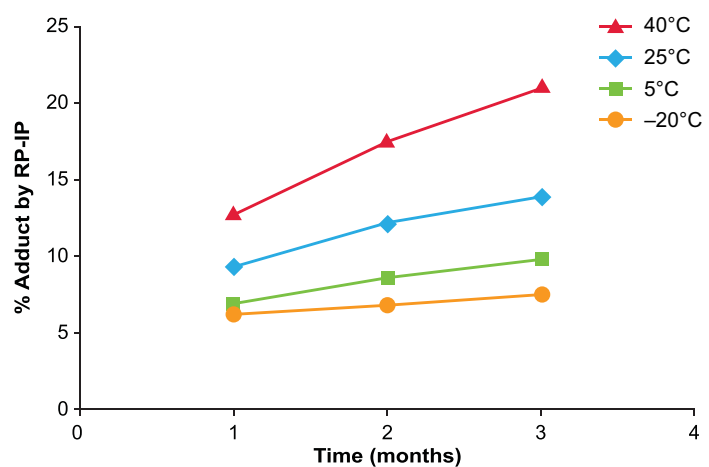

Fig. 1 Identification of LP in formulated mRNA-LNP by RP-IP HPLC. a Reversed phase-ion pair high performance liquid chromatography (RP-IP HPLC) provides high resolution mRNA length-based separations to assess content and quality of mRNA products, as shown by the separation of 6 mRNAs of different lengths $(659,785,914,1106,2498$, and 2993 nucleotides) in a single lipid nanoparticle (LNP) formulation across retention times of 9.5-15 min. Peaks prior to $9.5 \mathrm{~min}$ are degradants and the peaks at $22 \mathrm{~min}$ form part of the column wash. b The RP-IP HPLC analysis of pure mRNA (blue) yields a single MP (retention time $15 \mathrm{~min}$ ), with shorter degradation products and impurities eluting prior (retention time, 10-14.5 min), whereas mRNA extracted from an mRNA-LNP formulation (black) yields an additional late-eluting peak region (LP; retention time 19-21 min). The UV spectrum at each peak apex obtained from an on-line 3D UV detector shows an identical profile with maximum absorbance of $260 \mathrm{~nm}$ (inset). c CE analysis of extracted mRNA shows a single peak, with no additional late-eluting species. $\mathbf{d}$ LP species in mRNA extracted from an mRNA-LNP formulation can be further resolved with adjusted gradient conditions and show a polydisperse fingerprint of species. e An mRNA-LNP formulation was stored for 3 months at 4 different temperatures $\left(40^{\circ} \mathrm{C}\right.$, red; $25^{\circ} \mathrm{C}$, blue; $5^{\circ} \mathrm{C}$, green; $-20^{\circ} \mathrm{C}$, orange), and sampled at 1,2 , and 3 months for analysis by RP-IP HPLC; each data point is a single incubation condition run in a single RP-IP HPLC assay. Representative data are shown. AU, absorption units.

protonated monoisotopic mass of cytosine. The neutral mass loss of $132.03 \mathrm{Da}$ corresponds to the monoisotopic residue mass of ribose $^{26}$, indicating that ribose is unmodified. The cytosine adduct formation serves as only one example; across several lipid systems studied, a variety of lipid adducts were observed, with reactions across all 4 mRNA nucleobases demonstrated in model systems (Supplementary Fig. 6).

Reaction modeling to identify contributors to adduct formation. To investigate the source of above-mentioned reactions, combinations of mRNA with individual LNP components (ionizable cationic lipid, polyethylene glycol [PEG] lipid, sterol, and phosphocholine) were examined. LP was only observed in preparations with the ionizable lipid component, with no impact from other lipids (Fig. 4a). When the RP-IP HPLC chromatogram of a binary combination of mRNA and ionizable lipid was compared to that of mRNA-LNP formulated with the same ionizable lipid, an identical LP peak profile was observed by RPIP (Fig. 4b), suggesting the same reactions were occurring in this simplified system. 
a

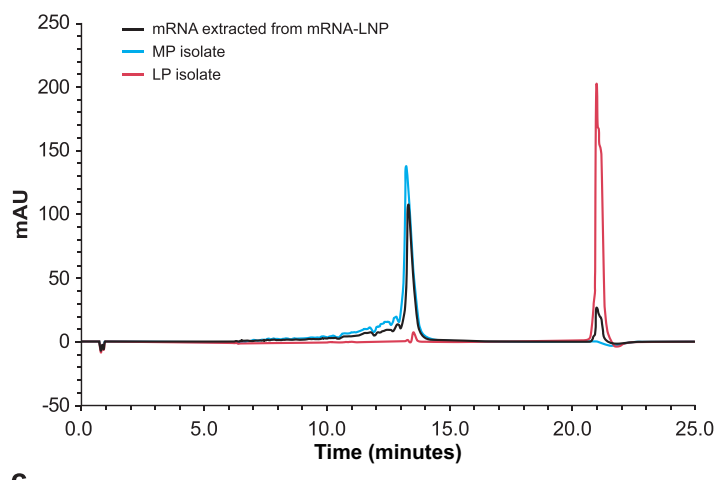

C

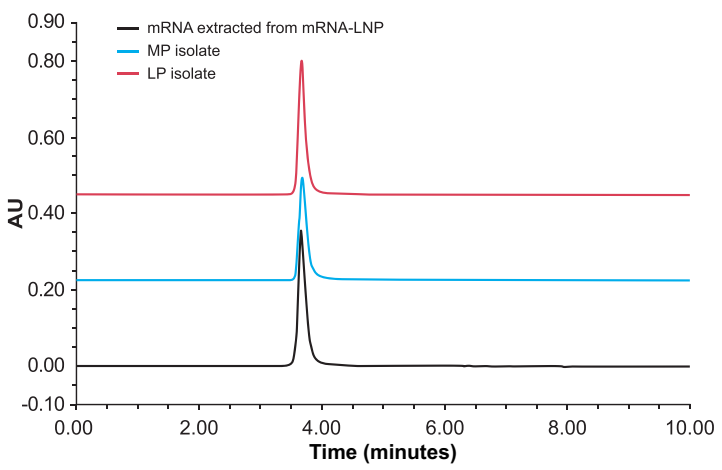

b

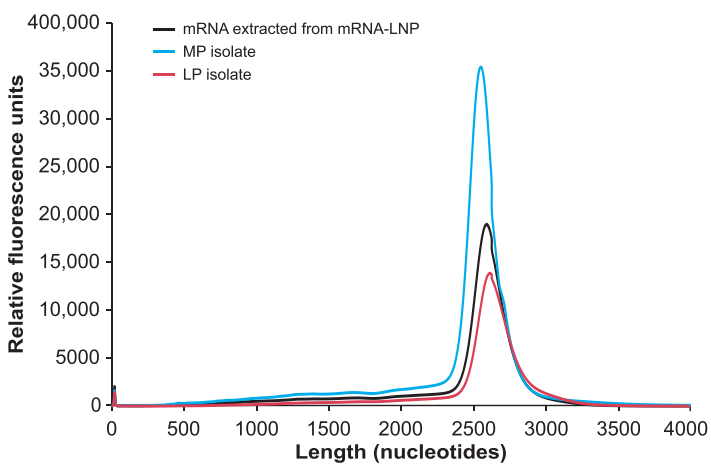

Fig. 2 Second dimension intact analysis of isolated MP and LP. a The reversed phase-ion pair high performance liquid chromatography (RP-IP HPLC) chromatogram of mRNA extracted from the mRNA-lipid nanoparticle (mRNA-LNP, black) is overlaid with RP-IP HPLC re-analysis of isolated mRNA main peak (MP, blue) and late eluting-peak (LP, red), showing preserved retention time of each isolated region. $\mathbf{b}$ The capillary electrophoresis (CE) electropherogram of mRNA extracted from the mRNA-LNP (black) is overlaid with electropherograms of the isolated MP (blue) and LP (red), showing no difference in migration time. c The size-exclusion chromatography (SEC) chromatograms of mRNA extracted from the mRNA-LNP (black) is overlaid with SEC chromatograms of isolated MP (blue) and LP (red), showing no presence of aggregation in either fraction. Representative data from 4 repeat experiments are shown. $\mathrm{AU}$, absorption units.

Additional ionizable lipid chemistries $(>100)$ were next examined, all of which generated quantifiable levels of mRNAlipid adduct, demonstrating a broad class effect. The RP-IP HPLC profile of these adduct species varied in retention times across different chemistries likely based on size and hydrophobicity of the adducted lipid structure (Supplementary Fig. 7). Across different lots of ionizable lipid tested in the binary system, there were variations in relevant abundance of a consistent set of LP species (Fig. 4c), suggesting these reactions are driven by impurities of the ionizable lipid rather than chemical reactivity of the lipid itself. Subsequently, a highly reactive ionizable lipid from the chemistry screen was studied to understand the chromatographic behavior of intact adducted mRNA. At 1 day, a discrete shift in retention produced LP peaks at 10-12 min; increased tailing from 12.5-22.5 min was observed as the MP was depleted (Fig. 4d). This observation strongly suggests a single adduct event can shift the RNA molecule to LP, and accumulation of multiple adducts per mRNA molecule further drives increases in retention time.

The binary system was then used to study contributions of the mRNA molecule to adduct formation. A series of mRNA sequences ranging in length from 700 to $>4000$ nucleotides in length was assessed at equivalent masses in individual binary reactions, resulting in increasing LP with mRNA length (Fig. 4e). As relative UV intensity of the LP peak in the RP-IP HPLC assay is correlated with relative mass, this observation is consistent with a constant rate of reaction on the single base level resulting in greater mass shifts to LP with increasing sequence length. This correlation highlights the relevance of these reactions for mRNA as a high molecular weight nucleic acid polymer. Although comparable lipid systems are used in low molecular weight RNA products such as small interfering RNA (siRNA), the same molar reaction rate observed in mRNA systems would result in lower levels of adduct formation of siRNA on an intact mass basis due to the stochastic nature of adduct formation. Notably, the same adduct peaks can be observed across mRNAs of different lengths in the chromatographic profile with a decrease in retention time of each LP region with increasing mRNA length (Fig. 4f). We can understand this as an average hydrophobicity of the adducted mRNA sequence; as sequence length increases, the same adducts comprise less of the total molecule, lowering the impact on retention. The combined impact of mRNA length and lipid lot is shown in Fig. 4g, demonstrating that the simplified binary system can be used as a predictive model for the final LNP.

Oxidative impurities of ionizable lipid as a driver of adduct formation. To further understand the implicated role of ionizable lipid impurities as the driver of adduct formation (Fig. $4 a-c$ ), impurities and degradants that are common to the ionizable amino-lipid family such as those shown in Fig. 5a were next examined. $\mathrm{N}$-oxide formation is a common degradation pathway for tertiary amine-containing molecules under oxidative stress ${ }^{27}$. Although relatively stable, $\mathrm{N}$-oxide can further hydrolyze to secondary amines and aldehyde counterparts (Fig. 5b), reported typically through metal catalysis ${ }^{28}$. Since transition metals are well-controlled in mRNA-LNP raw materials due to their propensity to accelerate mRNA degradation, the relevance of $\mathrm{N}$-oxide hydrolysis in the absence of metal catalysts was 
a

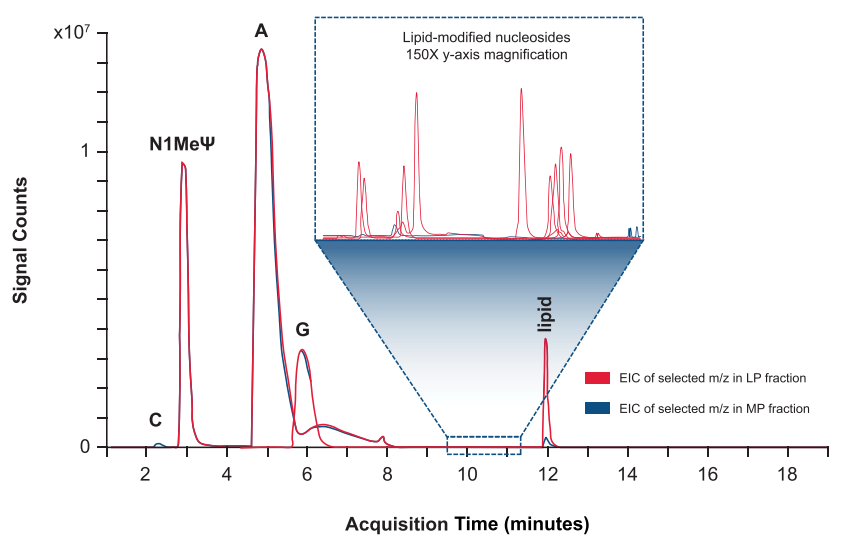

b
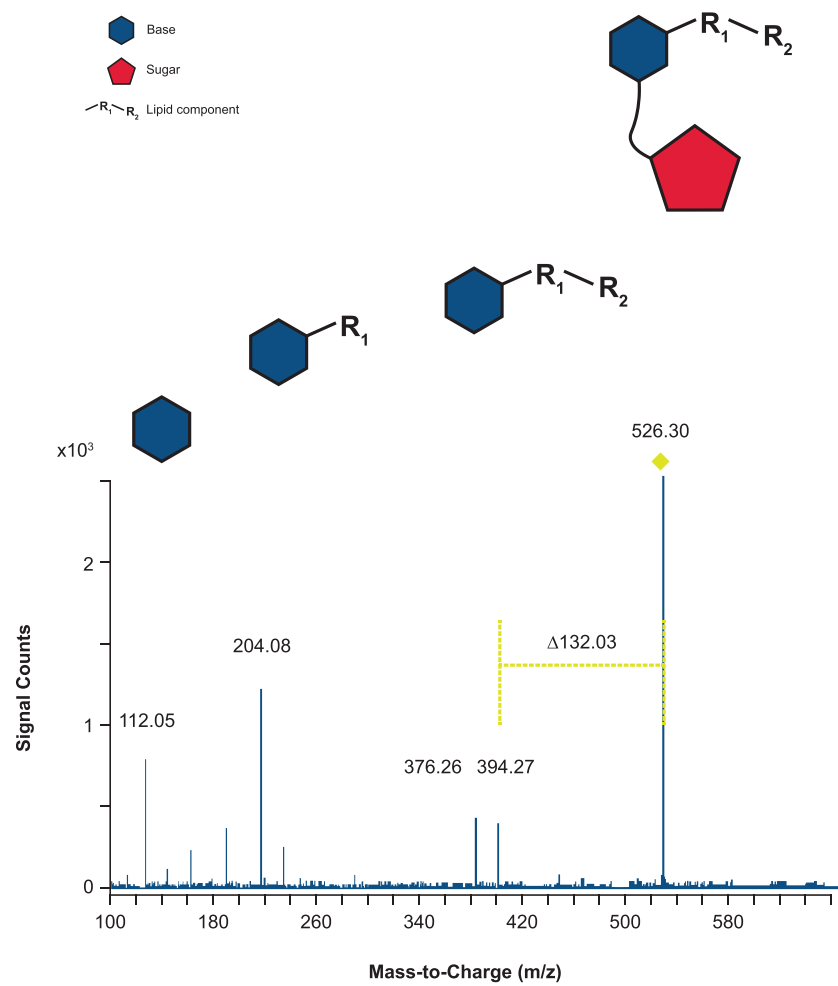

evaluated. N-oxide standard was generated from a representative ionizable lipid, precipitated in acidic buffer, and analyzed by reversed phase-ultra-high-performance liquid chromatography with charged aerosol detection (RP-UPLC-CAD) and MS/MS detection. Three peaks generated under these conditions at retention time $8.7,10.3$, and 25 min were identified by MS/MS as the 3 secondary amines resulting from hydrolysis of $\mathrm{N}$-oxide. To detect the residual mass, the degraded $\mathrm{N}$-oxide solution was derivatized with an aminooxy-PEG to label all aldehyde functionalities. Unique peaks at 9.5 and 16 min were detected by RPUPLC-CAD, corresponding to aldehyde products of $\mathrm{N}$-oxide hydrolysis; the third expected aldehyde peak likely elutes in the column void (Fig. 5c). These studies demonstrate that even in relatively mild acidic conditions, $\mathrm{N}$-oxide hydrolysis can generate secondary amines and aldehydes without the use of metallic catalysts. Further analysis revealed that the presence of $\mathrm{N}$-oxide leads to high levels of LP, with almost complete conversion of the mRNA to LP within 3 days in the binary system (Fig. 5d).
Fig. 3 Identification of single modified nucleosides by LC/MS/MS. a Isolated mRNA main peak (MP) and late eluting-peak (LP) were enzymatically digested to single nucleosides and analyzed by liquid chromatography with tandem mass spectrometry (LC/MS/MS). Extracted ion chromatograms (EICs) of selected mass-to-charge ratios $(\mathrm{m} / \mathrm{z}$ ) corresponding to various unmodified nucleosides, lipid-modified nucleosides, and carry-over lipid in LP (red) and MP mRNA (blue) fractions are overlaid. The selected $\mathrm{m} / \mathrm{z}$ for EICs include unmodified nucleosides (cytidine [C; $2.3 \mathrm{~min}$ ], N1-methylpseudouridine [N1-MelJ.l; $3 \mathrm{~min}$ ], adenosine $[A ; 5 \mathrm{~min}]$, guanosine $[\mathrm{G} ; 6 \mathrm{~min}])$, lipid (11.9 $\mathrm{min})$, and several lipid-modified nucleosides (9.5-11.5 min) observed in LP mRNA fractions. $150 x y$-axis zoom of various low-abundant lipid-modified nucleosides eluting between 9.5 and $11 \mathrm{~min}$ is shown in the inset. $\mathbf{b}$ The precursor ion at $\mathrm{m} / \mathrm{z} 526.30$ was isolated and subjected to collision-induced dissociation. Based on fragmentation pattern, the original nucleoside was determined to be cytidine, covalently modified by one chain of the ionizable lipid prepared in the binary. The fragment ion of $\mathrm{m} / \mathrm{z} 112.05$ corresponds to the exact mass of protonated cytosine (nucleobase). The characteristic neutral mass loss of $132.03 \mathrm{Da}$, corresponds to the monoisotopic residue mass of ribose $^{26}$. The fragment ion of $\mathrm{m} / \mathrm{z} 394.27$ corresponds to the lipid-modified cytosine, which further undergoes fragmentation to $\mathrm{m} / \mathrm{z} 376.26$ (loss of water) and 204.08 (at the internal ester), confirming the identity of the lipid chain. This cytidine modification is provided as a representative example, but similar characteristic neutral mass losses of 132.03 were observed for lipid modifications across all 4 nucleobases. Representative data from 4 repeat experiments are shown.

Investigation of mRNA adduction by aldehydes. Covalent modification of DNA by aldehydes has been previously reported $^{29}$, setting a precedent for the reactivity of newly identified aldehydes from N-oxide degradation of amino lipids. Two representative aldehydes from the $\mathrm{N}$-oxide degradation pathway of an ionizable lipid (a linear, 17-carbon chain and a branched, 25-carbon chain) were studied. The aldehydes were individually spiked into ionizable lipids as impurities prior to precipitation in binary reactions. Significant increases in lipid-mRNA adduct levels were observed with increasing spike concentration for both aldehydes. By RP-IP HPLC, peaks corresponding to mRNA adducts with the smaller linear aldehyde elute at $7.5 \mathrm{~min}$ (Fig. 5e) and those for the larger branched aldehyde elute at 8-10 min (Fig. 5f). When mRNA from the linear aldehyde binary was subjected to enzymatic digestion and positive mode LC-MS/MS analysis, there was a corresponding increase in 2 resulting nucleoside masses of $\mathrm{m} / \mathrm{z} 526.3$ (Fig. 5g) and 540.3 (Fig. 5h) which were further elucidated by MS/MS as being products of aldehyde addition to cytidine (Fig. 3b). As described in Fig. 4d, the increase in tailing at the highest spike concentration in the intact mRNA chromatography is potentially due to the accumulation of multiple lipid adducts per mRNA strand, contrasting the initial discrete shift that occurs for each aldehyde with a single adduct addition. Although hydrolysis of N-oxide is one particularly relevant pathway for the generation of aldehydes in the LNP system, similar species may be present as lipid raw material impurities or oxidative degradants. These could generate the diversity of adduct species observed within the intact mRNA and single nucleoside level.

Impact of lipid-mRNA adduct on protein expression and product shelf life. The role of chemical modifications on protein expression was explored. MP and LP fractions were isolated by RP-IP HPLC from 2 mRNA formulations encoding the human erythropoietin (hEPO) protein and analyzed for in vitro protein expression in BJ fibroblasts. Expression of hEPO protein was comparable between the isolated MP, the unfractionated 
a

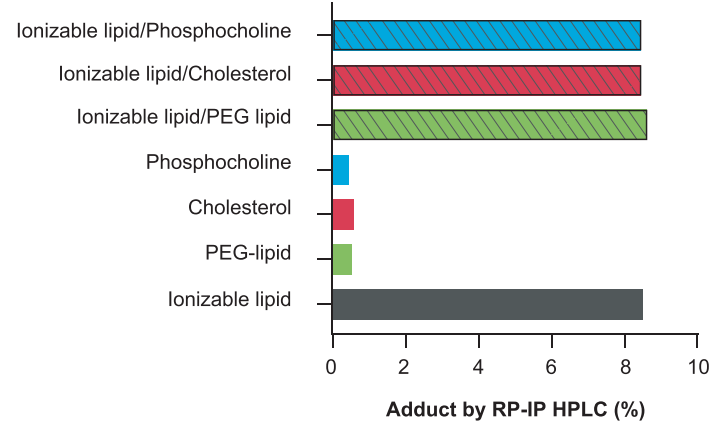

C

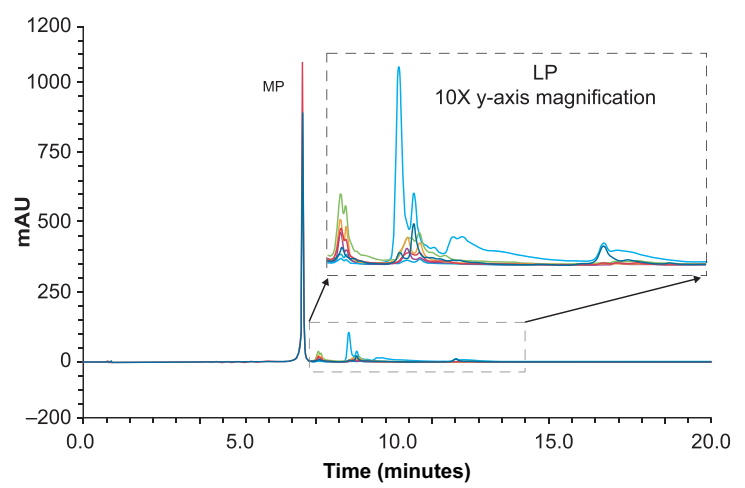

e

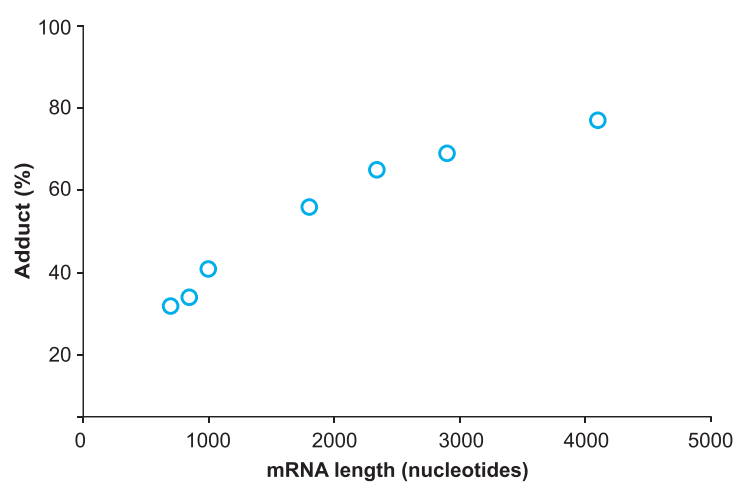

b

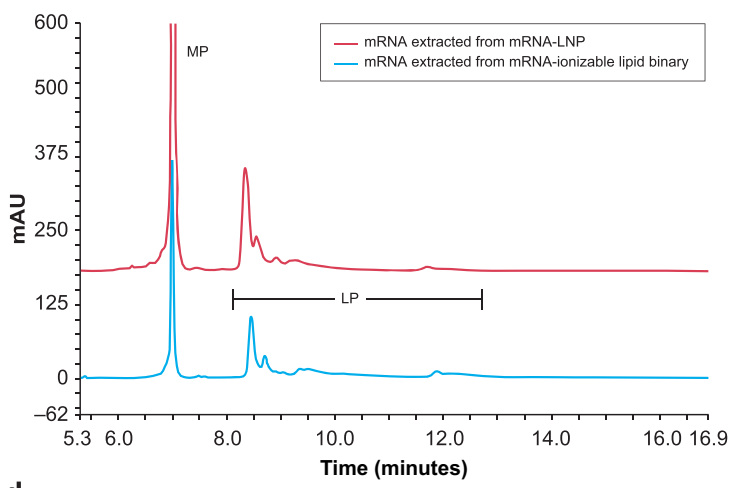

d

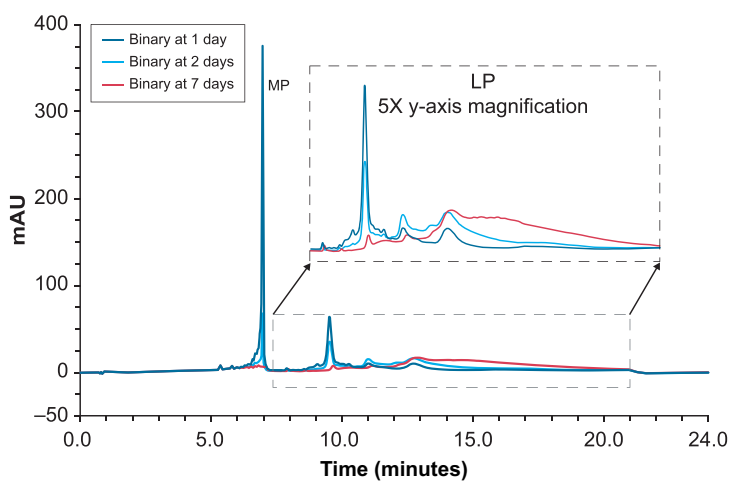

f

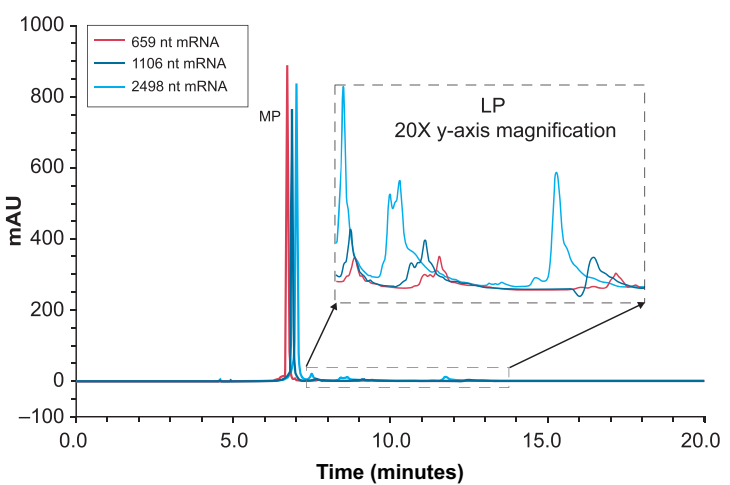

g

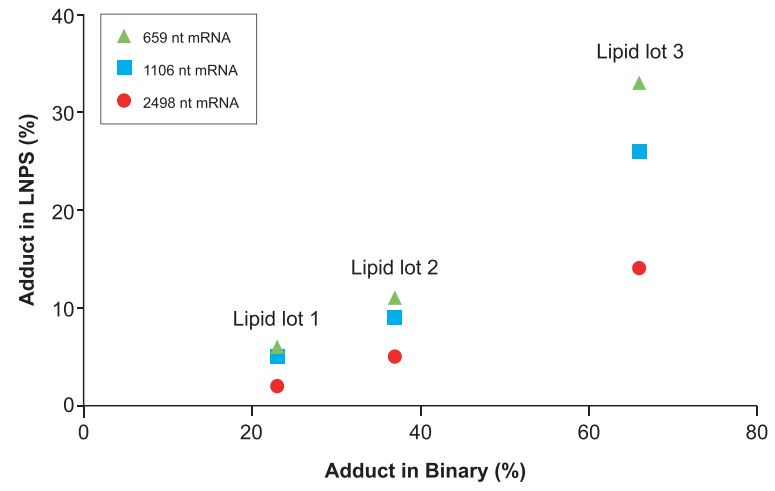

extracted mRNA, and hEPO assay control (Fig. 6a); in contrast, very low protein expression was detected from the isolated LP (Fig. 6a). Separately, 5 LNP formulations were subjected to various levels of degradation, after which the mRNA was extracted from the LNP and analyzed by RP-IP HPLC, CE, and for in vitro protein expression in HeLa cells (Fig. 6b). When mRNA integrity was assessed using RP-IP HPLC, a correlation between relative mRNA integrity (including the adduct as an impurity) and protein expression was found (Fig. 6b). In contrast, mRNA integrity measured by CE only weakly correlated with protein expression; all samples had a narrow range of relative purity despite the decrease in protein expression (Fig. 6b). Extrapolating these 
Fig. 4 Contribution of mRNA and lipid to adduct formation. a Binary reactions of single lipid components shows that only combinations including the ionizable lipid resulted in significant adduct formation with mRNA by reversed phase-ion pair high performance liquid chromatography (RP-IP HPLC). PEG, polyethylene glycol. b The RP-IP HPLC adduct profiles of mRNA extracted from the binary system (blue) and mRNA-lipid nanoparticle (mRNA-LNP, red) with the same ionizable lipid show the same qualitative peak profile. mRNA main peak (MP). c Seven different lots of the same ionizable lipid were prepared in binaries with mRNA, yielding a variety of peak profiles and abundances of adduct species in the overlaid RP-IP HPLC chromatograms. d Adduct formation in a binary reaction with a highly reactive lipid was evaluated by RP-IP HPLC at 1 day (black), 2 days (blue), and 7 days (red), showing increased tailing likely corresponding to the accumulation of multiple adducts per mRNA molecule. e Adduct formation in binary reactions was evaluated with mRNA molecules of different lengths by RP-IP HPLC at equivalent mRNA masses. An increase in late eluting-peak (LP) with length is consistent with a constant rate of modification on the single nucleotide level. f RP-IP HPLC chromatographs of 659 (red), 1106 (dark blue), and 2498-nucleotide (blue) mRNAs show an increase and left shift of each adduct peak with increasing mRNA length. $\mathbf{g}$ Adduct formation as a function of mRNA length was assessed by RP-IP HPLC for mRNA-LNP and corresponding binaries. A positive correlation was observed, with more adduct at longer mRNA lengths. Representative data from $\geq 3$ repeat experiments are shown. $\mathrm{AU}$, absorbance units.

correlations shows a total loss in protein expression despite mRNA purity $>60 \%$ by CE. These data demonstrate the potential of such adduct reactions to reduce activity of mRNA-LNP products and the inadequacy of CE to determine mRNA quality in LNP formulations.

The introduction of impurities into the vaccine manufacturing process could potentially impact vaccine stability even under refrigerated conditions. The loss of mRNA purity to adduct formation under refrigeration $\left(5^{\circ} \mathrm{C}\right)$ was examined for 2 vaccine formulations utilizing the same mRNA sequence and lipid system. One vaccine had poor controls in place to limit adduct formation (Vaccine A) and the other had rigid controls (Vaccine B; Fig. 6c). Data for Vaccine A show an initial delta of $15 \%$, indicating rapid adduct formation during product processing or prior to testing. This is followed by substantial loss in mRNA integrity by almost $50 \%$ to adduct formation over 3 months in refrigeration. By contrast, Vaccine B demonstrated comparatively low levels of adduct formation with a negligible increase over time (Fig. 6c).

\section{Discussion}

LNP-based nucleic acid delivery systems enabled the development and emergency use authorization of 2 COVID-19 mRNA vaccines at unprecedented speed. The agility offered by the mRNA-LNP platform promises a new era in vaccine and drug development. Ionizable lipids are an essential component of the LNP; they aid in encapsulating mRNA into the LNP system, define the biophysical profile and stability, and determine pharmacological performance of the vaccine or therapeutic.

Herein we report identification of a lipid-modified class of mRNA impurities generated by electrophilic degradants and impurities originating in the ionizable lipid; these impurities disrupt mRNA translation and negatively impact the activity of LNP-formulated mRNA products. Additionally, such impurities are difficult to identify using traditional techniques used to assess mRNA integrity in mRNA-LNP such as CE. RP-IP HPLC provides specificity and sensitivity to detect adducted mRNA molecules, which are otherwise difficult to identify due to the low rate of modification. Indeed, data suggest that RP-IP HPLC can detect even single adduct events on intact mRNA, whereas in contrast, even purified LP is not distinguished by the conventional CE methodology. Furthermore, it is critical that formation of these mRNA-lipid adducts are examined during formulation design, clinical evaluation, and commercialization by using appropriate RP-IP HPLC methodologies to ensure there is no loss in mRNA integrity.

A potential source of impurities is through hydrolysis of $\mathrm{N}$-oxide to aldehydes; this reaction is broadly relevant to tertiary amines commonly used in LNP formulation of siRNA and mRNA $^{28,30}$. It remains highly probable that formation of this class of adducts has been missed by historically applied analytical technologies such as $\mathrm{CE}$, thus overlooking an important critical quality attribute of mRNA-LNP. This represents a gap in quality control of mRNA-LNPs during manufacturing, particularly as it pertains to consistency and activity of the resultant drug product. In this study, we report the ability of RP-IP HPLC to resolve aldehyde species differing only by a short alkyl chain, highlighting the selectivity of the RP-IP HPLC method to detect minute differences on the intact mRNA molecule.

The pathway reported herein identifies an important class of impurities that may be present in all lipid-based mRNA systems. These reactions can be mitigated through raw material control, manufacturing process parameters, formulation design, and LNP storage conditions. It thus remains critical to monitor and control lipid adduct formation during the research, development, and manufacture of LNP-formulated nucleic acid products. Importantly, this study highlights the need for advanced methods such as RP-IP HPLC to ensure the quality, consistency, and efficacy of any pharmaceutical product.

\section{Methods and materials}

Formulations. All mRNA and LNP formulations used in this work were representative of GMP-grade material. Several mRNA molecules were used throughout this work; all were 5-prime capped, 3-prime poly-adenylated sequences with modified uridine chemistry (N1-methyl-pseudouridine) encoding different vaccine targets and other proteins.

RNA extraction from mRNA-LNPs and binaries. mRNA was extracted from the mRNA-LNP formulation or lipid binary mixture by isopropanol precipitation. $100 \mu \mathrm{L}$ of mRNA-LNP or binary was diluted 10 -fold in $900 \mu \mathrm{L}$ ammonium acetate $(60 \mathrm{mM})$ in isopropanol, vortexed briefly, and centrifuged at $14,000 \mathrm{~g}$ for $15 \mathrm{~min}$ at $4{ }^{\circ} \mathrm{C}$. The supernatant was discarded and the pellet was washed with $1 \mathrm{~mL}$ isopropanol, vortexed, and centrifuged at $4{ }^{\circ} \mathrm{C}$; the pellet was dried in vacuo and resuspended in $100 \mu \mathrm{L}$ RNase-free water at room temperature.

Reversed phase ion pair chromatography (RP-IP). Sample separation was performed on a DNAPac RP column with $4-\mu \mathrm{m}$ particles and dimensions of $2.1 \times 100 \mathrm{~mm}$ (Thermo Fisher Scientific) at a flow rate of $0.35 \mathrm{~mL} / \mathrm{minute}$ and column temperature of $65^{\circ} \mathrm{C}$. Mobile phase A consisted of dibutylammonium acetate $(50 \mathrm{mM}$; TCI America) and triethylammonium acetate $(100 \mathrm{mM}$; SigmaAldrich) and mobile phase B consisted of 50\% acetonitrile (Sigma-Aldrich), dibutylammonium acetate $(50 \mathrm{mM})$, and triethylammonium acetate $(100 \mathrm{mM})$. Separation was accomplished by step-gradient with an initial 1.5 -minute hold at $25 \%$ B, a 3.0-minute gradient from $25-50 \%$ B, a 14.5 -minute gradient from $50-56 \%$ $\mathrm{B}$, and a 0.5 -minute gradient and hold at $100 \% \mathrm{~B}$. Modified gradient conditions for LP resolution were performed with an initial 1.5-minute hold at $25 \% \mathrm{~B}$, a 1 -minute gradient from $25-45 \% \mathrm{~B}$, a 12.5 -minute gradient from $45-100 \% \mathrm{~B}$, and a 0.5 minute gradient and hold at $100 \% \mathrm{~B}$. Approximately $2 \mu \mathrm{g}$ of mRNA were injected. mRNA was detected by UV at $260 \mathrm{~nm}$. LP is quantified as the relative percent of the total chromatographic peak area.

Capillary electrophoresis (CE). Sample separation was performed on a Fragmen Analyzer (Agilent Technologies), an automated multiplexed CE system equipped with an LED light source and charged-coupled device detector. RNA was quantitatively and qualitatively analyzed using the RNA Analysis Kit (Agilent Technologies DNF-489-0500). The RNA separation gel was mixed with an intercalating dye (AATI) at a v/v ratio of 10,000:1 for use as the separation matrix. RNA was 
a

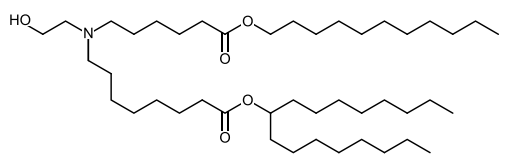

heptadecan-9-yl 8-((2-hydroxyethyl)(6-oxo-6-(undecyloxy)hexyl)amino)octanoate

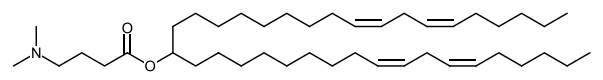

(6E,8E,28E,31E)-heptatriaconta-6,8,28,31-tetraen-19-yl 4-(dimethylamino)butanoate

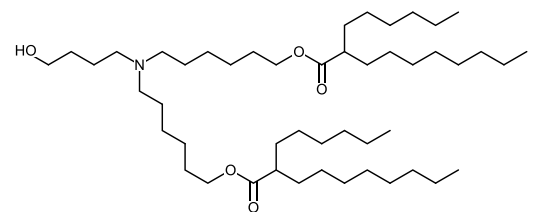

((4-hydroxybutyl)azanediyl)bis(hexane-6,1-diyl) bis(2-hexyldecanoate)

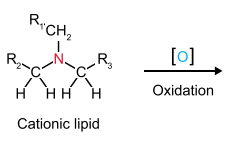<smiles>[R]CN(O)C([R])([2H])C(C)C</smiles>

Cationic lipid $\mathrm{N}$-oxide

H-B 1 : B

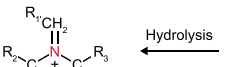

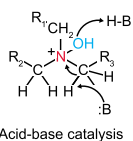

$\mathrm{R}^{\mathrm{R}} \mathrm{CH}^{2}$

$\mathrm{R}_{2} \rightarrow \mathrm{C}=\mathrm{N}-\mathrm{R}_{3}$

H C $_{H}=\mathrm{B}$

Hemi-aminal

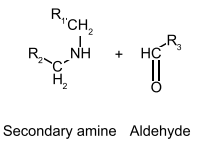

c

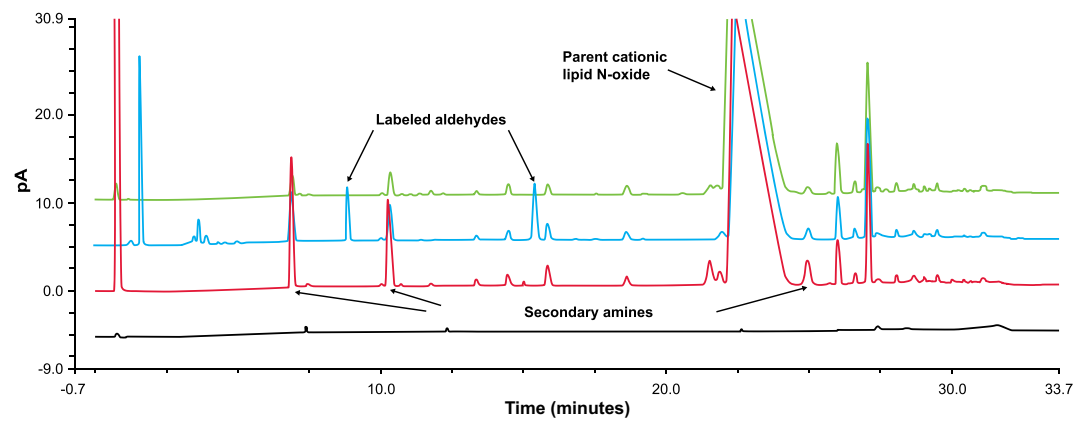

d

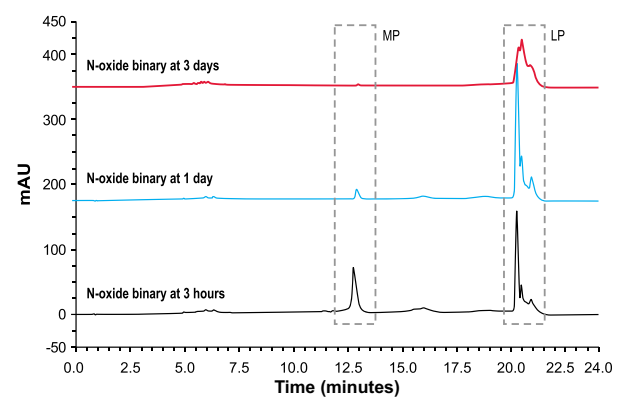

$\mathbf{f}$

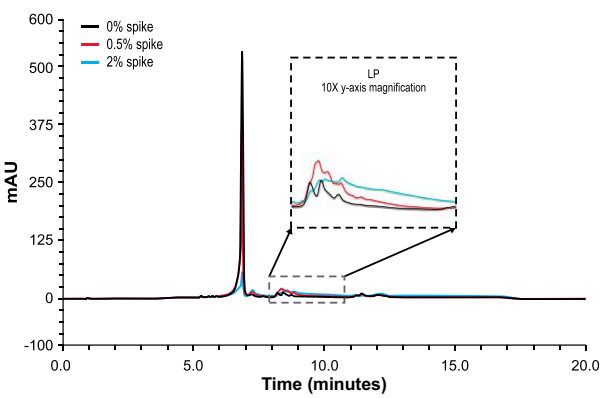

e

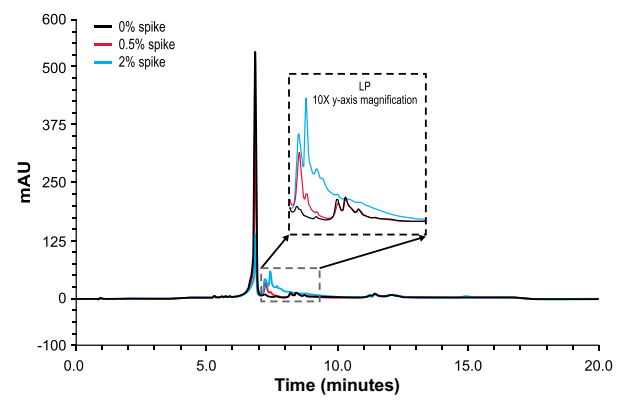

h

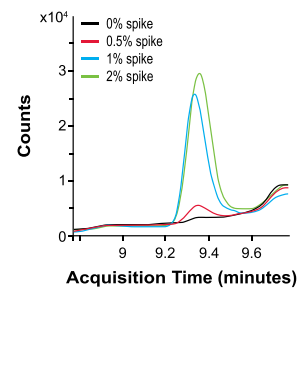

denatured at $70{ }^{\circ} \mathrm{C}$ for $2 \mathrm{~min}$ and cooled on ice prior to analysis. Denatured RNA samples were electrokinetically injected at $5 \mathrm{kV}$ for $6 \mathrm{~s}$, and electrophoresis was performed for $40 \mathrm{~min}$ at $8 \mathrm{kV}$. An RNA ladder (AATI) was similarly analyzed as a calibrator for nucleotide size. Data were analyzed using PROSize 2.0 software (Agilent Technologies).
Size exclusion chromatography (SEC). Sample separation was performed on a Zenix SEC-300 $150 \times 4.6 \mathrm{~mm}$ protein SEC column (Sepax) on a Waters H-Class UPLC (Waters). The mobile phase condition was $100 \mathrm{mM}$ Tris acetate $/ 2.5 \mathrm{mM}$ EDTA pH 8 with an isocratic flow of $0.25 \mathrm{~mL} /$ minute and UV detection at $260 \mathrm{~nm}$. 
Fig. $5 \mathbf{~ N}$-oxide as a driver of adduct formation. a Tertiary-amine-containing ionizable lipids currently used in RNA-lipid nanoparticle (RNA-LNP) products. b $\mathrm{N}$-oxide forms through tertiary amine oxidation and undergoes an acid/base-catalyzed hydrolysis at the amine to generate aldehydes and secondary amines. c N-oxide acid hydrolysis products were detected by reversed phase-ultra-high-performance liquid chromatography with charged aerosol detection and tandem mass spectrometry (RP-UPLC-CAD MS/MS); N-oxide standard (green), acid-precipitated N-oxide standard with (blue) and without (red) aminooxy-polyethylene glycol (aminooxy-PEG) label, and buffer baseline (black). Secondary amines from N-oxide hydrolysis elute at 8.7, 10.3, and $25 \mathrm{~min}$. Corresponding aminooxy-PEG-derivatized aldehydes elute at 9.5 and $16 \mathrm{~min}$, with the third likely in the column void. pA, pico ampere. $\mathbf{d}$ Binary reactions of $m R N A$ with pure $\mathrm{N}$-oxide result in high late eluting-peak (LP). RP-IP analysis of mRNA extracted from the binary is shown at $3 \mathrm{~h}$ (black), 1 day (blue), and 3 days (red) MP, mRNA main peak. e Binary reactions spiked with pure 17-carbon aldehyde and $\mathbf{f}$ pure 25-carbon aldehyde products from the $\mathrm{N}$-oxide degradation pathway result in high levels of mRNA modification. Reversed phase-ion pair high performance liquid chromatography (RP-IP HPLC) chromatograms of the extracted mRNA are shown with no aldehyde spike (black), $0.5 \%$ aldehyde (red), and $2 \%$ aldehyde (green). $\mathbf{g}$, $\mathbf{h}$ Binary reactions with the pure 17-carbon aldehyde from the $\mathrm{N}$-oxide degradation pathway were analyzed for single nucleoside modifications. Binaries were prepared with no aldehyde (black), $0.5 \%$ aldehyde (red), $1 \%$ aldehyde (blue), and $2 \%$ aldehyde (green) spike. mRNA was extracted, enzyme-digested, and analyzed by liquid chromatography with tandem mass spectrometry (LC-MS/MS). The mass-to-charge ratios ( $\mathrm{m} / \mathrm{z}$ ) corresponding to aldehyde-cytidine adducts ( $\mathrm{m} / \mathrm{z}$ 526.3 and 540.3) increased with aldehyde spike level. A representative ionizable lipid system, heptadecan-9-yl 8-((2-hydroxyethyl)(6-oxo-6-(undecyloxy) hexyl)amino)octanoate is used here. Representative data from $\geq 3$ repeat experiments are shown.

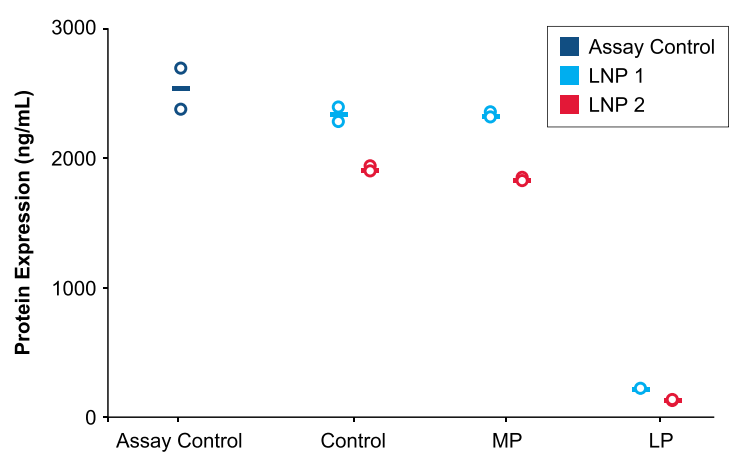

b

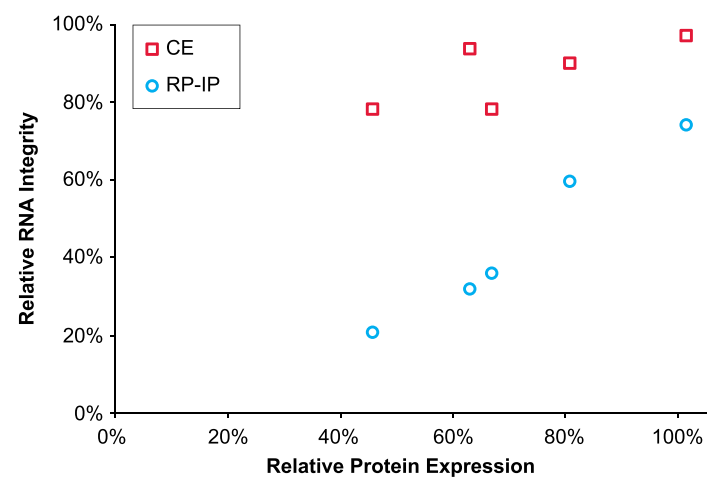

C

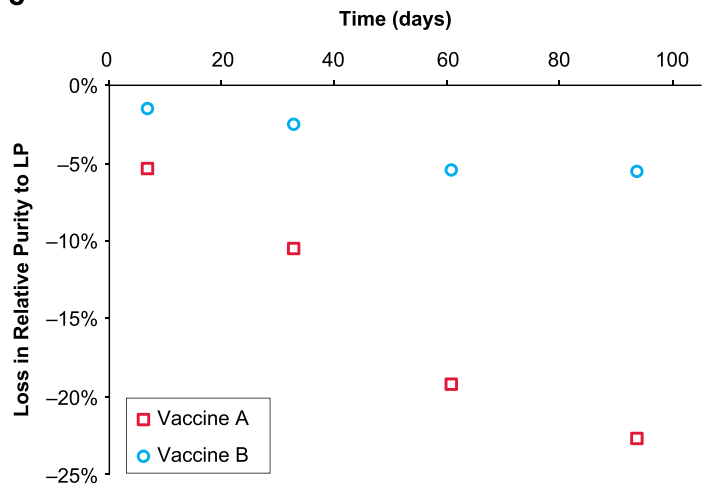

Fig. 6 mRNA adduction reduces protein expression. a Isolated main peak (MP) and late eluting-peak (LP) alongside control mRNA-lipid nanoparticle (mRNA-LNP) and an assay positive control were tested for in vitro protein expression in BJ fibroblasts after 48 h. RNA was extracted from 2 hEPO-LNP formulations by isopropanol precipitation and purified by RP-IP to generate MP and LP prior to transfection. The assay control is a pure hEPO mRNA standard, the control sample is mRNA extracted from each formulation prior to RP-IP separation, and the MP and LP are isolated fractions. Results from 2 replicate transfection wells is plotted with the mean. $\mathbf{b}$ Five different mRNA-LNP samples were prepared using different ionizable lipids and incubated at $5^{\circ} \mathrm{C}$ to generate varying levels of adduct and degradation. RNA was extracted from the mRNA-LNP by isopropanol precipitation and evaluated by reversed phase-ion pair (RP-IP), capillary electrophoresis (CE), and in vitro protein expression in HeLa cells as measured by mean fluorescence intensity. Relative expression as a percentage of the neat mRNA expression is plotted versus relative integrity as a percentage of the neat mRNA integrity, determined by relative area using both CE and RP-IP HPLC. c Loss in mRNA purity to adduct formation in 2 vaccine formulations is plotted over 3 months at refrigerated conditions. Poor process control led to high LP in Vaccine A, but adduct was well-controlled in Vaccine B. Representative data are shown for 3 repeat experiments for Fig. $6 a$ and multiple repeat experiments for Fig. $6 \mathrm{~b}$ and c.

Binary model preparation. mRNA-lipid binaries were formed by mixture of mRNA and ionizable lipid. Unless otherwise noted, a standard 2000-nucleotide mRNA $(0.135 \mathrm{mg} / \mathrm{mL})$ was prepared in sodium acetate $(37.5 \mathrm{mM} ; \mathrm{pH} 5.3$; Sigma Aldrich) and mixed at a 3:1 ratio with an ionizable lipid solution $(4 \mathrm{mg} / \mathrm{mL})$ in ethanol, followed by incubation at room temperature for $24 \mathrm{~h}$. RNA was extracted from the binary using isopropanol precipitation as described above prior to further analysis.
Enzymatic digestion of mRNA to ribonucleosides. Total nuclease digestion was performed as previously described ${ }^{31}$ with modifications. mRNA was incubated with 15 units of benzonase (Millipore), 2 units of phosphodiesterase I (SigmaAldrich), and 1.3 units of quick calf intestinal alkaline phosphatase (New England Biolabs) in buffer containing Tris- $\mathrm{HCl}$ (Invitrogen), $\mathrm{NaCl}$, and $\mathrm{MgCl}_{2}$ (Invitrogen) at $37^{\circ} \mathrm{C}$ for $2 \mathrm{~h}$. 


\section{Positive mode liquid chromatography mass spectrometry (LC-MS/MS)}

Nucleosides were separated in an increasing water/acetonitrile gradient containing $0.1 \%$ formic acid at $0.4 \mathrm{~mL} /$ minute on an Accucore C30 column with $2.6 \mu \mathrm{m}$ particles and dimensions of $2.1 \times 250 \mathrm{~mm}$ (Thermo Scientific) at $50^{\circ} \mathrm{C}$. Ultraviolet detection of nucleosides was monitored at $260 \mathrm{nM}$. Mass spectral data were acquired on an Agilent 6530 QTOF (Agilent Technologies) in positive electrospray ionization (ESI) mode. The mass range was $100-2000 \mathrm{~m} / \mathrm{z}$, drying gas temperature was $290^{\circ} \mathrm{C}$ at a flow rate of $11 \mathrm{~L} / \mathrm{min}$, and the nebulizer gas pressure was $35 \mathrm{psi}$. The capillary, fragmentor, and octupole voltage were set at 3500,100 , and $750 \mathrm{~V}$, respectively. External calibration was used for accurate mass measurement. For tandem mass spectrometry (MS/MS), precursor ions were subjected to collisioninduced dissociation (CID) and MS/MS fragmentation analysis.

Tandem mass spectrometry (MS/MS). MS/MS experiments using CID were performed following RP-HPLC separation. Acquisition of a full mass scan was followed by targeted MS/MS scans of precursor ions of interest. Data were acquired on an Agilent 6530 QTOF (Agilent Technologies) for MS/MS analysis. Normalized collision energy for ion activation was 30 arbitrary units. Narrow ion isolation width $(\sim 1.3 \mathrm{~m} / \mathrm{z})$ was used for isolation.

$\mathbf{N}$-oxide precipitation and labeling. An $\mathrm{N}$-oxide standard of ionizable lipid was generated. The N-oxide compound was dissolved in ethanol at $4 \mathrm{mg} / \mathrm{mL}$. One milliliter of the $\mathrm{N}$-oxide solution was added dropwise to $3 \mathrm{~mL}$ of sodium acetate (37.5 mM; pH 5.3; Sigma Aldrich), followed by incubation at room temperature for $24 \mathrm{~h}$. Prior to LC-CAD-MS analysis, aminooxy-PEG ( $1 \mathrm{mM}$; Thermo Fisher) was used as a labeling agent to detect and identify aldehydes present in the system.

Aldehyde spike preparation. Synthetic aldehyde standards corresponding to the 17 -carbon linear chain and 25-carbon branched chain of a representative ionizable lipid (heptadecan-9-yl 8-((2-hydroxyethyl)(6-oxo-6-(undecyloxy)hexyl)amino) octanoate) were generated. Aldehyde solutions $(4 \mathrm{mg} / \mathrm{mL})$ were individually prepared in ethanol and mixed with an ionizable lipid solution $(4 \mathrm{mg} / \mathrm{mL})$ in ethanol to various target compositions, listed in \% w/w of the aldehyde to ionizable lipid. Binary preparations, intact mRNA HPLC analyses, and digested nucleoside LC/MS analyses were then performed as described above.

\section{Reversed phase ultra-performance liquid chromatography with charged} aerosol detection (RP-UPLC-CAD). LNP formulations were diluted in ethanol and the supernatant was analyzed. Lipid standards were separately diluted and analyzed to identify and quantify lipid components. RP-UPLC separation was performed on an ACE Excel 2 Super C18 column (Advanced Chromatography Technologies) with $2.1 \times 150 \mathrm{~mm}$ dimensions heated to $60^{\circ} \mathrm{C}$. Mobile phase A consisted of $0.1 \%$ trifluoroacetic acid (TFA) (Thermo Fisher Scientific) in water and mobile phase B consisted of 60/40/0.1\% isopropyl alcohol/tetrahydrofuran/ TFA (Thermo Fisher Scientific). Lipids were eluted by step-gradient with an initial 1.5-minute hold at 5\% B, a 4.5-minute gradient from 5-48\% B and 4-minute hold, a 1-minute gradient from $48-56 \% \mathrm{~B}$ and 12 -minute hold, and an 8-minute gradient from $56-96 \%$ B and 2-minute hold. Lipids were detected by CAD using an evaporator temperature of $35^{\circ} \mathrm{C}$ and analytical gas regulation mode.

Protein expression in BJ fibroblasts. BJ fibroblasts (ATCC; catalog \#CRL-2522) were cultured in complete media (Eagle's minimum essential medium [EMEM] with L-glutamine; $10 \%$ FBS). Cells were seeded in 96-well plates (20,000 cells/well) $24 \mathrm{~h}$ prior to transfection; extracted mRNA was transfected with Lipofectamine L2000 (Life Technologies, catalog \#11668019) at $250 \mathrm{ng} / \mathrm{well}$ and incubated for $48 \mathrm{~h}$ at $37^{\circ} \mathrm{C}$ before collection of the supernatant for analysis. An hEPO mRNA standard was included as an assay control.

Detection of protein expression by flow cytometry. In vitro expression was performed in HeLa cells (ATCC, catalog \#CCL-2). Cells were seeded in Minimal essential medium (MEM) with 10\% FBS, 1X Glutamax, and 1X sodium pyruvate at $37^{\circ} \mathrm{C}$ at 15,000 cells in a total volume of $100 \mu \mathrm{L}$ per well and grown for $24 \mathrm{~h}$. Extracted mRNA was transfected with Lipofectamine L2000 (Life Technologies, catalog \#11668019) at $1333 \mathrm{ng}$ of mRNA per well and incubated for $18-20 \mathrm{~h}$ at $37^{\circ} \mathrm{C}$. To detect intracellular protein expression, cells were first fixed and permeabilized (Cytoxfix/Cytoperm; BD Biosciences catalog \#554714) and then stained with a proprietary primary antibody (1/2000 dilution of $1 \mathrm{mg} / \mathrm{mL}$ stock). Data were acquired on the BD LSRFortessa ${ }^{\text {tx }}$ Flow Cytometer (BD Biosciences) using BD FACSDiva ${ }^{\text {mo }}$ version 8.0 software (BD Biosciences) and analyzed using FlowJo software version 10.4 (BD Biosciences). Mean fluorescence intensity was normalized using a neat mRNA positive control and non-translating negative control. The gating strategy can be found in Supplementary Fig. 8 .

Statistics and reproducibility. Assay measurements were taken from distinct samples. This is a descriptive study and as such no formal statistical analyses were performed. Experiments conducted for the identification and characterization of the MP and LP utilizing RP-IP HPLC, CE analysis of adduct-containing mRNA, resolution of the adduct peaks with UV spectral data, and increases in adduct at elevated storage temperature of the LNP (Fig. 1) acts as a representative example of analyses that have been performed repeatedly ( $>100$ times) with consistent reproducible findings. Experiments conducted for the isolation of adducts and analysis utilizing RP-IP, SEC and CE (Fig. 2) were replicated 4 times with similar results. Identification of modified nucleosides present in the adduct by nucleoside digest, differential analysis, and subsequent structural elucidation by MS/MS (Fig. 3) was replicated 4 times with similar results. For identifying the contribution of mRNA and lipids to adduct formation utilizing binary analysis of individual lipids, comparisons of ionizable lipid binary adduct results with the LNP, and qualitative observations in the binary with mRNA length and lipid lot (Fig. 4) have each been replicated $\geq 3$ times with similar results. Experiments conducted to determine the influence of $\mathrm{N}$-oxides in adduct formation utilizing the decomposition of N-oxide in acidic buffer, mRNA binaries with N-oxide, and RP-IP and MS analysis of aldehyde-spiked binaries (Fig. 5) have been replicated $\geq 3$ times with similar results. Experiments to determine the expression of the isolated adduct (Fig. 6a) have been replicated 3 times and experiments to determine loss in expression with adduct, and increase in adduct over storage across formulations (Fig. $6 \mathrm{~b}$ and $\mathrm{c}$ ) have been replicated $\geq 3$ times.

For data shown in the supplemental information, FTIR analysis (Supplement Fig. 2) was performed once. Illumina NGS (Supplemental Fig. 3), T1 digest followed by LC/UV/MS (Supplemental Fig. 4), and $\mathrm{NaOH}$ digest followed by RP-IP LC-UV (Supplemental Fig. 5) were replicated 2 times with similar results. Analysis of homopolymers in the binary system (Supplement Fig. 6) was performed once. Comparisons of adduct profiles (Supplement Fig. 7) have been generated with $>100$ lipid chemistries with similar results; 7 representative chromatograms are displayed.

Reporting summary. Further information on research design is available in the Nature Research Reporting Summary linked to this article.

\section{Data availability}

The authors declare that the data supporting the findings of this study are available within this Article and its Supplementary Information. The data that support the findings of this study are available from the corresponding authors upon reasonable request.

Received: 22 June 2021; Accepted: 28 September 2021; Published online: 22 November 2021

\section{References}

1. Gómez-Aguado, I. et al. Nanomedicines to deliver mRNA: state of the art and future perspectives. Nanomaterials 10, 364 (2020).

2. Kowalski, P. S., Rudra, A., Miao, L. \& Anderson, D. G. Delivering the messenger: advances in technologies for therapeutic mRNA delivery. Mol. Ther. 27, 710-728 (2019).

3. Baden, L. R. et al. Efficacy and safety of the mRNA-1273 SARS-CoV-2 vaccine. N. Engl. J. Med 384, 403-416 (2021).

4. Polack, F. P. et al. Safety and efficacy of the BNT162b2 mRNA Covid-19 vaccine. N. Engl. J. Med 383, 2603-2615 (2020).

5. Houseley, J. \& Tollervey, D. The many pathways of RNA degradation. Cell 136, 763-776 (2009).

6. Crommelin, D. J. A., Anchordoquy, T. J., Volkin, D. B., Jiskoot, W. \& Mastrobattista, E. Addressing the cold reality of mRNA vaccine stability. $J$. Pharm. Sci. 110, 997-1001 (2021).

7. Reichmuth, A. M., Oberli, M. A., Jaklenec, A., Langer, R. \& Blankschtein, D. mRNA vaccine delivery using lipid nanoparticles. Ther. Deliv. 7, 319-334 (2016)

8. Khurana, A. et al. Role of nanotechnology behind the success of mRNA vaccines for COVID-19. Nano Today 38, 101142 (2021).

9. Gilleron, J. et al. Image-based analysis of lipid nanoparticle-mediated siRNA delivery, intracellular trafficking and endosomal escape. Nat. Biotechnol. 31, 638-646 (2013).

10. Cullis, P. R. \& Hope, M. J. Lipid nanoparticle systems for enabling gene therapies. Mol. Ther. 25, 1467-1475 (2017).

11. Guevara, M. L., Persano, F. \& Persano, S. Advances in lipid nanoparticles for mRNA-based cancer immunotherapy. Front Chem. 8, 589959 (2020).

12. Huang, X. \& Chau, Y. Enhanced delivery of siRNA to retinal ganglion cells by intravitreal lipid nanoparticles of positive charge. Mol. Pharm. 18, 377-385 (2021).

13. Zhang, R. et al. Helper lipid structure influences protein adsorption and delivery of lipid nanoparticles to spleen and liver. Biomater. Sci. 9, 1449-1463 (2021). 
14. Liu, S. et al. Membrane-destabilizing ionizable phospholipids for organselective mRNA delivery and CRISPR-Cas gene editing. Nat. Mater. 20, 701-710 (2021).

15. Heyes, J., Palmer, L., Bremner, K. \& MacLachlan, I. Cationic lipid saturation influences intracellular delivery of encapsulated nucleic acids. J. Control Release 107, 276-287 (2005).

16. Sabnis, S. et al. A novel amino lipid series for mRNA delivery: improved endosomal escape and sustained pharmacology and safety in non-human primates. Mol. Ther. 26, 1509-1519 (2018)

17. Kim, M. et al. Engineered ionizable lipid nanoparticles for targeted delivery of RNA therapeutics into different types of cells in the liver. Sci. Adv. 7, eabf4398 (2021).

18. Prieve, M. G. et al. Targeted mRNA therapy for ornithine transcarbamylase deficiency. Mol. Ther. 26, 801-813 (2018).

19. Fenton, O. S. et al. Bioinspired alkenyl amino alcohol ionizable lipid materials for highly potent in vivo mRNA delivery. Adv. Mater. 28, 2939-2943 (2016).

20. Pogocki, D. \& Schoneich, C. Chemical stability of nucleic acid-derived drugs. J. Pharm. Sci. 89, 443-456 (2000).

21. Kanavarioti, A. HPLC methods for purity evaluation of man-made singlestranded RNAs. Sci. Rep. 9, 1019 (2019).

22. Azarani, A. \& Hecker, K. H. RNA analysis by ion-pair reversed-phase high performance liquid chromatography. Nucleic Acids Res 29, E7 (2001).

23. Wang, P., Akula, R., Chen, M. \& Legaspi, K. AN1616: SEC-MALS Method for Characterizing mRNA Biophysical Attributes. WYATT Technology. https:// wyattfiles.s3-us-west-2.amazonaws.com/literature/app-notes/sec-malsbiopolymers/AN1616-SEC-MALS-method-for-characterizing-mRNA.pdf (Date accessed: 28 May 2021).

24. Levin, D. S., Shepperd, B. T. \& Gruenloh, C. J. Combining ion pairing agents for enhanced analysis of oligonucleotide therapeutics by reversed phase-ion pairing ultra performance liquid chromatography (UPLC). J. Chromatogr. B Anal. Technol. Biomed. Life Sci. 879, 1587-1595 (2011).

25. Yang, M. H. \& Schaich, K. M. Factors affecting DNA damage caused by lipid hydroperoxides and aldehydes. Free Radic. Biol. Med 20, 225-236 (1996).

26. Takeshita, T. \& Kanaly, R. A. In vitro DNA/RNA adductomics to confirm DNA damage caused by benzo[a]pyrene in the Hep G2 cell line. Front Chem. 7, 491 (2019).

27. Baumeister, T. et al. Continuous flow synthesis of amine oxides by oxidation of tertiary amines. React. Chem. Eng. 4, 1270-1276 (2019).

28. Kok, G. B. \& Scammells, P. J. N-demethylation of N-methyl alkaloids with ferrocene. Bioorg. Med Chem. Lett. 20, 4499-4502 (2010).

29. Garaycoechea, J. I. et al. Alcohol and endogenous aldehydes damage chromosomes and mutate stem cells. Nature 553, 171-177 (2018).

30. Wu, Z. \& Li, T. Nanoparticle-mediated cytoplasmic delivery of messenger RNA vaccines: challenges and future perspectives. Pharm. Res. 38, 473-478 (2021).

31. Nelson, J. et al. Impact of mRNA chemistry and manufacturing process on innate immune activation. Sci. Adv. 6, eaaz6893 (2020).

\section{Acknowledgements}

We thank Melissa Moore, Orn Almarsson, Don Parsons, Huijuan Li, Shaila Jayaram, and Will Issa for their intellectual input and support, and Ed Miracco for assistance with the preparation of this manuscript. Jack Kramarczyk and Beth Lally led formulation efforts to apply findings to the LNP. Amelie Dricot-Ziter and Kamaljeet Sandhu supported in vitro expression assays. Dave Mauger performed next-generation sequencing analysis, and Christopher Tunkey and Christopher Pepin assisted with preparation of the nextgeneration sequencing data. Tao Jiang, Srujan Gandham, and Serenus Hua provided support on LC/MS analyses, nucleoside digestion, and differential analysis approaches, and Greg Mercer and Jin Lim provided N-oxide and aldehyde standards. Medical writing and editorial assistance was provided by Wynand van Losenoord and Srividya Ramachandran, PhD, of MEDiSTRAVA in accordance with Good Publication Practice (GPP3) guidelines, funded by Moderna Inc, and under the direction of the authors.

\section{Author contributions}

M.P. and D.G. contributed equally to this manuscript. M.P. identified covalent lipid addition and developed and performed RP-IP and other intact mRNA analyses, preparative mRNA fractionation, and binary studies. D.G. identified the N-oxide pathway and performed binary studies, lipid analyses, and $\mathrm{N}$-oxide and aldehyde analysis. R.Y. designed and carried out LC/MS and MS/MS experiments for modified nucleoside structural analysis. J.S. made initial observation on the modified mRNA and provided technical expertise to N-oxide work. P.W. provided leadership and direction throughout the project.

\section{Competing interests}

All authors are employees of and shareholders in Moderna Inc. The authors declare no other competing interests.

\section{Additional information}

Supplementary information The online version contains supplementary material available at https://doi.org/10.1038/s41467-021-26926-0.

Correspondence and requests for materials should be addressed to Phil White.

Peer review information Nature Communications thanks Balasubrahmanyam Addepalli and Xucheng Hou for their contribution to the peer review of this work.

Reprints and permission information is available at http://www.nature.com/reprints

Publisher's note Springer Nature remains neutral with regard to jurisdictional claims in published maps and institutional affiliations.

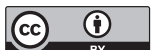

Open Access This article is licensed under a Creative Commons Attribution 4.0 International License, which permits use, sharing, adaptation, distribution and reproduction in any medium or format, as long as you give appropriate credit to the original author(s) and the source, provide a link to the Creative Commons license, and indicate if changes were made. The images or other third party material in this article are included in the article's Creative Commons license, unless indicated otherwise in a credit line to the material. If material is not included in the article's Creative Commons license and your intended use is not permitted by statutory regulation or exceeds the permitted use, you will need to obtain permission directly from the copyright holder. To view a copy of this license, visit http://creativecommons.org/ licenses/by/4.0/.

(C) The Author(s) 2021 